\title{
Quadratic Optimization in Ill-Posed Problems
}

\author{
F. Ben Belgacem* $\quad$ S.-M. Kaber ${ }^{\dagger}$
}

\begin{abstract}
Ill posed quadratic optimization frequently occurs in control and inverse problems and are not covered by the Lax-Milgram-Riesz theory. Typically small changes in the input data can produce very large oscillations on the output. We investigate the conditions under which the minimum value of the cost function is finite and we explore the 'hidden connection' between the optimization problem and the least-squares method. Eventually, we address some examples coming from optimal control and data completion, showing how relevant our contribution is in the knowledge of what happens for various ill-posed problems. The results we state bring a substantial improvement to the analysis of the regularization methods applied to the ill-posed quadratic optimization problems.
\end{abstract}

KEYWORDS: Quadratic optimization, least-squares, ill-posedness, Picard's principle, optimal control, data completion.

\section{Introduction}

Let $H$ be a real Hilbert space with infinite dimension, $(\cdot, \cdot)$ denotes the inner product and $\|\cdot\|$ is the associated norm. Assume that $A$ is a continuous and semi-definite linear operator on $H$. For a given $b$ in $H$, we consider the following unconstrained semi-definite quadratic optimization problem

$$
\gamma_{J}=\min _{y \in H}\left(\frac{1}{2}(A y, y)-(b, y)\right)=\min _{x \in H} J(y) .
$$

Without restriction of the generality $A$ may be taken symmetric. When it is coercive, that is $(A y, y) \geq \alpha\|y\|^{2}$, for all $y \in H$, and $\alpha$ is a given positive real number, the Riesz-Lax-Milgram theory ensures the well-posedness of the problem (1). The argument of the minimum does exist, specified by the equation $A x=b$, is unique and depends continuously on $b$ (see [7] for instance). The operator $A$ is therefore invertible with a continuous inverse on $H$. In addition, the function $J(\cdot)$ can be put under a least squares form. Suffices it to notice that the square root of $A$, denoted by $\sqrt{A}$, is well defined (see [26]), is elliptic and invertible. Then, there exists $d \in H$ such that $b=\sqrt{A} d$ and we have that

$$
J(y)=\frac{1}{2}\|\sqrt{A} y-d\|^{2}-\frac{1}{2}\|d\|^{2} .
$$

*LMAC, EA 2222, Université de Technologie de Compiègne, Centre de Recherches de Royallieu, BP 20529, 60205 COMPIEGNE Cedex, FRANCE (faker.ben-belgacem@utc.fr).

${ }^{\dagger}$ LJLL (UMR CNRS 7598), Université Pierre \& Marie Curie, B.C. 187, 4 place Jussieu, 75252 PARIS Cedex 05, FRANCE. (kaber@ann.jussieu.fr). 
Let us observe that writing $J(\cdot)$ under a least-squares form has no practical interest for wellposed problems.

There is a rich mathematical and programming material on semi-definite quadratic optimization in finite dimensions (see $[6,30,18]$ for instance and [12] for a wide bibliography). Semi-definite operators $A$ with a non-closed range arise more difficulties and things are less formalized and seem less achieved. Whilst coercive quadratic optimization problems are conclusively handled (see, eg, [7]), the semi-definite optimization problems, ill-posed in the Hadamard sense (see [13]), require some more work. Filling in this blank is worth considering. Because of its consequences on regularization strategies we may use for the quadratic optimization problem (1), the main point we focus on is the condition under which (1) can be put under a least-squares form. That condition turns to be the simple fact that the functional $J(\cdot)$ be bounded from below.

We have been led to consider that optimization problem because of our interest to the data completion process. The variational framework for the corresponding Cauchy problem, recently led down in [4] is stated to be equivalent to problem (1), with $A$ a semi-definite Hilbert-Schmidt operator. Similar ill-posed problems come from so many fields of the physics and the mathematics, from astronomy, optics, geophysics, optimal filtering, statistics ..., etc. Moreover, ill-posed integral equations defined by regular kernels are current in image processing, in data completion, in optimal control, in machine learning, and generally in many technological and industrial processes (see, e.g., [19, 20, 25]).

The outline of the paper is as follows. In section 2, we investigate the condition under which $\gamma_{J}$ is finite and state that the cost function $J(\cdot)$ has a least-squares form. In Section 3, we compare two methods to solve the ill-posed problem $A x=b$. One consists in minimizing $J(\cdot)$ and the other is the least squares procedure minimizing the residual $\|b-A x\|$. We check why the first approach is better suited since some spurious modes are exhibited that are brought by the least-squares. These modes are damped in the minimization of $J(\cdot)$. Explaining what happen when $A$ is unbounded is the subject of Section 4 while Section 5 is an illustration of our results in the particular case of a separable space $H$ and a compact operator $A$. Finally, Section 6 is devoted to some interesting examples coming from the optimal control, controllability and the inverse problems of data completion.

Some notions and notations. - Most often the adjoint operator of $A$ is denoted by $A^{*}, \mathcal{N}(A)$ and $\mathcal{R}(A)$ stand for the kernel and the range of $A$ respectively. Recall that $\mathcal{N}\left(A^{*}\right)^{\perp}=\overline{\mathcal{R}(A)}$ and $\mathcal{N}(A)^{\perp}=\overline{\mathcal{R}\left(A^{*}\right)}$. The symbol $F^{\perp}$ is for the orthogonal set of $F$ and $\bar{F}$ is for the topological closure of $F$. When $A$ is continuous, symmetric and semi-definite with a non-closed range, its spectrum is a compact set included in $[0,\|A\|]$ and contains 0 which is an accumulation point of it. The spectral decomposition theorem is at the basis of the functional calculus on $A$ (see $[26,8,7])$. We are able define the powers $\left(A^{\theta}\right)_{\theta \geq 0}$ of $A$ and we have $A^{\theta} A^{\tau}=A^{\theta+\tau}$ for any 
$\theta, \tau \geq 0$ (see $[26,8])$. It holds that

$$
(A y, y)=\|\sqrt{A} y\|^{2}, \quad \forall y \in H .
$$

\section{Quadratic Optimization with Bounded Operators}

Let us observe that whenever the kernel of $A$ is not trivial, that is $\mathcal{N}(A) \neq\{0\}$, the data $b$ that are worth to look at are those orthogonal to $\mathcal{N}(A)\left(b \in \mathcal{N}(A)^{\perp}\right)$ otherwise $\gamma_{J}$ is obviously infinite $\left(\gamma_{J}=-\infty\right)$. The optimization problem can then be restricted to the Hilbert space $\overline{\mathcal{R}(A)}$. With these respects, assuming that $\mathcal{N}(A)=\{0\}$ does not restrict the generality. We focus only on the operators to which the Lax-Milgram lemma fails to apply, $A$ is therefore non-coercive. We deduce that (see $[7,1])$

(i.) the range $\mathcal{R}(A)$ is not closed and is dense in $H$. (ii.) the functional $J(\cdot)$ is strictly convex.

When $b \in \mathcal{R}(A)$ and then $b=A x$, the optimization problem (1) has $x$ as the only solution with $\gamma_{J}=J(x)$ (see [9]). Now, if $b \notin \mathcal{R}(A)$ can the quadratic functional $J(\cdot)$ be bounded from below? The answer is: yes, it can! More specifically, we have

Theorem 2.1 The minimum value $\gamma_{J}$ of the functional $J(\cdot)$ is finite if and only if $b \in \mathcal{R}(\sqrt{A})$.

Since $\mathcal{R}(\sqrt{A})$ is not closed and is dense in $H$. A small perturbation on $b \in \mathcal{R}(\sqrt{A})$ would make $J(\cdot)$ be unbounded from below. The optimization problem is therefore ill-posed. The proof of this theorem requires two preliminary lemmas.

Lemma 2.2 Assume that $\gamma_{J}$ is finite. Then, we have

$$
\langle b, y\rangle \leq\left(\sqrt{-2 \gamma_{J}}\right)\|\sqrt{A} y\|, \quad \forall y \in H .
$$

Proof. That $J(\cdot)$ is minimized on $H$ with the infimum value $\gamma_{J}$ yields that

$$
t^{2}(A y, y)-2 t(b, y)-2 \gamma_{J} \geq 0, \quad \forall y \in H, \forall t \in \mathbb{R} .
$$

Putting it under the canonical form, we obtain that

$$
(A y, y)\left(t-\frac{(b, y)}{(A y, y)}\right)^{2}-\left(\frac{(b, y)^{2}}{(A y, y)}+2 \gamma_{J}\right) \geq 0, \quad \forall y \in H, y \neq 0, \forall t \in \mathbb{R} .
$$

For such an inequality to hold, it is necessary to have that

$$
(b, y) \leq \sqrt{-2 \gamma_{J}(A y, y)}=\left(\sqrt{-2 \gamma_{J}}\right)\|\sqrt{A} y\|, \quad \forall y \in H .
$$

The proof is complete. 
Lemma 2.3 Let $T$ be a bounded operator on $H$, with $\mathcal{N}\left(T^{*}\right)=\{0\}$, and $b \in H$. Assume that for a given real-number $\eta \geq 0$, we have that

$$
(b, y) \leq \eta\|T y\|, \quad \forall y \in H .
$$

Then, there exists $d \in H$ such that $b=T^{*} d$.

Proof. Notice that estimate (2) yields in particular that $b \in \mathcal{N}(T)^{\perp}$. For any $y \in \mathcal{R}(T)$, let $z \in \overline{\mathcal{R}\left(T^{*}\right)}$ so that $y=T z$. Such a $z$ is unique. Introduce the linear form $\ell(\cdot)$ as follows

$$
\ell(y)=(b, z), \quad \forall y \in \mathcal{R}(T) .
$$

On account of (2), we obtain the boundedness

$$
\ell(y) \leq \eta\|T z\|=\eta\|y\|, \quad \forall y \in \mathcal{R}(T) .
$$

This implies that $\ell(\cdot)$ can be continuously extended to $H$. Indeed, because $\overline{\mathcal{R}(T)}=\mathcal{N}\left(T^{*}\right)^{\perp}=$ $\{0\}^{\perp}$, the range $\mathcal{R}(T)$ is dense in $H$. By the Riesz theorem there exists $d \in H$ such that

$$
\ell(y)=(d, y), \quad \forall y \in \mathcal{R}(T)
$$

We deduce that

$$
(b, z)=(d, y)=(d, T z)=\left(T^{*} d, z\right), \quad \forall z \in \mathcal{R}\left(T^{*}\right),
$$

which yields that $\left(b-T^{*} d\right) \in \mathcal{N}(T)$. Given that $b$ and $T^{*} d$ are both in $\mathcal{N}(T)^{\perp}$, we derive that $b-T^{*} d=0$. The proof is achieved.

Proof of Theorem 2.1. Assume that $b=\sqrt{A} d$ with $d \in H$. Since $\mathcal{R}(\sqrt{A})$ is dense in $H$ we have then

$$
\min _{y \in H} J(y)=\min _{y \in H}\left(\frac{1}{2}\|\sqrt{A} y\|^{2}-(d, \sqrt{A} y)\right)=\min _{z \in H}\left(\frac{1}{2}\|z\|^{2}-(d, z)\right)=-\frac{1}{2}\|d\|^{2} .
$$

Therefore, $\gamma_{J}$ is finite.

Assume now that $\gamma_{J}$ is finite. Using Lemma 2.2 we obtain that

$$
(b, y) \leq\left(\sqrt{-2 \gamma_{J}}\right)\|\sqrt{A} y\|, \quad \forall y \in H .
$$

Now applying Lemma 2.3, with $T=\sqrt{A}$, there exists $d \in H$ such that $b=\sqrt{A} d$. The theorem is proved.

Remark 2.1 From Theorem 2.1, three situations are possible for the optimization problem. If $b \in \mathcal{R}(A)$, there is a unique $x \in H$ such that $\gamma_{J}=J(x)$, it is given by $A x=b$, else if $b \in \mathcal{R}(\sqrt{A}) \backslash \mathcal{R}(A)$ then $J(\cdot)$ is bounded from below, i.e., $\gamma_{J}>-\infty$ and the minimum is not attained and finally if $b \notin \mathcal{R}(\sqrt{A})$ we have that $\gamma_{J}=-\infty$.

Corollary 2.4 Problem (1) can be put under a least-squares form if and only of $J(\cdot)$ is bounded from below $\left(\gamma_{J}\right.$ is finite). 
Proof. When $\gamma_{J}$ is finite, we have by Theorem 2.1 that $b \in \mathcal{R}(\sqrt{A})$ and the function $J(\cdot)$ is given by

$$
J(y)=\frac{1}{2}\|\sqrt{A} y-d\|^{2}-\frac{1}{2}\|d\|^{2}, \quad \forall y \in H .
$$

The density of $\mathcal{R}(\sqrt{A})$ in $H$ yields that

$$
\gamma_{J}=-\frac{1}{2}\|d\|^{2}
$$

The proof is complete.

Remark 2.2 Be aware that these properties in the proof of Corollary 2.4 are but of a theoretical interest. Neither $\sqrt{A}$ nor $d$ are available to us. The data we really dispose of are $A$ and $b$ and reconstructing $\sqrt{A}$ or $d$ is a costly task and is out of record. However, as will be seen in the examples provided in Section 6, the value of $\gamma_{J}$ can be directly computed from the data which has tremendous effects on the regularization of problem (1).

Remark 2.3 The condition that $b \in \mathcal{R}(\sqrt{A})$ is nothing else than the general source condition (see $[23,16])$. In the regularization theory, such an assumption is made on the exact solution while it appears naturally on the data in our analysis.

\section{A Linear Inverse Problem}

Mathematically, a linear inverse problem is often expressed as finding $x \in H$ which solves the equation

$$
A x=b .
$$

$A$ is a linear operator with non-closed range. Assume that $A$ is injective for simplicity and that it is symmetric. If the data $b$ is not in the range of $A$ there is no solution to (3), so the inverse problem is generalized and we seek a solution $x$ to the optimization problem (1). This is, for instance, the way the Conjugate Gradient method works. Another class of methods is based on the minimization of the residual such as the GMRES, FOM or MINRES methods (see [27]). Each of them consists in minimizing the least squares functional

$$
L(y)=\frac{1}{2}\|A y-b\|^{2}=\frac{1}{2}\|\nabla J(y)\|^{2} .
$$

On account of the density of $\mathcal{R}(A)$ in $H$ the minimum value of $L(\cdot)$ is necessarily zero.

When $b \notin \mathcal{R}(\sqrt{A})$, there is no other possibility than the minimizing of the least-squares functions $L(\cdot)(J(\cdot)$ is not bounded from below. Otherwise, if $b \in \mathcal{R}(\sqrt{A}) \backslash \mathcal{R}(A)$, we pursue the comparison and the possible connections between the two classes of methods, those involved in the minimization of $J(\cdot)$ and those concerned with the minimization of $L(\cdot)$.

Lemma 3.1 Assume that $b \in \mathcal{R}(\sqrt{A})$. Let $\left(x_{n}\right)_{n} \subset H$ be a minimizing sequence of $J(\cdot)$. Then it is minimizing $L(\cdot)$. The sequence $\left(A x_{n}\right)_{n} \subset H$ converges toward $b$ and $\left(\sqrt{A} x_{n}\right)_{n} \subset H$ converges toward $d$. Furthermore, if $\left(x_{n}\right)_{n}$ is bounded then $b \in \mathcal{R}(A)$. 
Proof. $i$.- Let $\left(x_{n}\right)_{n} \subset H$ be a minimizing sequence of $J(\cdot)$ that is the limit of $\left(J\left(x_{n}\right)\right)_{n}$ is $\gamma_{J}>-\infty$. On account of Remark 2.2, we have that $\left(\sqrt{A} x_{n}\right)_{n} \subset H$ converges toward $d$. Thanks to the boundedness of $\sqrt{A}$, it comes out that $\left(A x_{n}\right)_{n} \subset H$ converges toward $b=\sqrt{A} d$. This achieve the first statements of the lemma.

ii.- Assume that $\left(x_{n}\right)_{n}$ is bounded then, modulo an extracted subsequence, it is weakly convergent toward $x \in H$. Since $A$ is linear and bounded then $\left(A x_{n}\right)_{n}$ weakly converges toward $A x$, which means that $A x=b$. As a result, we have that $b \in \mathcal{R}(A)$.

Lemma 3.2 Assume that $b \in \mathcal{R}(\sqrt{A})$. There exist a sequence $\left(x_{n}\right)_{n} \subset H$ such that $\left(A x_{n}\right)_{n} \subset H$ converges toward $b,\left(\left(L\left(x_{n}\right)\right)_{n \in \mathbb{N}}\right.$ converges toward 0$)$, which is not minimizing $J(\cdot)$.

Proof. The proof comes from the facts that the range $\mathcal{R}(\sqrt{A})$ is dense and that $\sqrt{A}$ has no bounded inverse. For any integer $n \geq 1$, there exists $x_{n} \in H$

$$
\left\|\sqrt{A} x_{n}-d\right\| \geq n\left\|\sqrt{A}\left(\sqrt{A} x_{n}-d\right)\right\|=n\left\|A x_{n}-b\right\| .
$$

Normalizing to $\left\|\sqrt{A} x_{n}-d\right\|=1$, we have that

$$
\left\|\nabla J\left(x_{n}\right)\right\|=\left\|A x_{n}-b\right\| \leq \frac{1}{n} .
$$

from which $\left(\nabla J\left(x_{n}\right)\right)_{n}$ converges toward zero and $\left(\left(L\left(x_{n}\right)\right)_{n \in \mathbb{N}}\right.$ converges toward 0 . In the other hand side, it is direct that for $n \geq 1$,

$$
J\left(x_{n}\right)=\frac{1}{2}-\frac{1}{2}\|d\|^{2} \neq \gamma_{J} .
$$

This achieves the proof.

Remark 3.1 Lemma 3.2 tells that $\left(\nabla J\left(x_{n}\right)\right)_{n}$ converges to zero does not necessarily imply that $\left(J\left(x_{n}\right)\right)_{n}$ converges toward $\gamma_{J}$. We say with Auslender that $J(\cdot)$ is not asymptotically wellbehaved (see [3]).

Remark 3.2 Any minimizing sequence $\left(x_{n}\right)_{n}$ of $L(\cdot)$ is such that $\left(A x_{n}\right)_{n}$ converges toward $b$, while $\left(\sqrt{A} x_{n}\right)_{n}$ may not converge toward $d$. This is far from being unimportant. Indeed, for some concrete problems, such as the data completion that will be investigated later on, the least squares method conceals some spurious modes that are filtered by the minimization of $J(\cdot)$. The effect of these observations on the regularization procedures is appreciable as will be discussed in the future.

\section{What about Unbounded Operators?}

Almost all the results stated for bounded operators can be extended as well to unbounded operators. For the others, only some slight modifications must be added, in particular for 
Lemma 2.3. Assume that $A$ is an unbounded operator on $H$, with a dense domain $D(A)$, selfadjoint and semi-definite. Consequently, $A$ is a closed operator (see [7]). Again, the functional calculus allows the construction of the operator $\sqrt{A}$, with a dense domain $D(\sqrt{A})$ (see $[26]$ ). We have that $D(A) \subset D(\sqrt{A})$. Moreover, since $\mathcal{N}(A)=\mathcal{N}(\sqrt{A})=\{0\}$, the subspaces $\mathcal{R}(A)$ and $\mathcal{R}(\sqrt{A})$ are dense in $H$ and $\mathcal{R}(A) \subset \mathcal{R}(\sqrt{A})$. In the unbounded case, the minimization problem (1) is changed to

$$
\min _{x \in D(A)} J(y)=\min _{y \in D(A)}\left(\frac{1}{2}(A y, y)-(b, y)\right)=\gamma_{J} .
$$

Analogous results as Theorem 2.1 for the new optimization problem are provided in

Theorem 4.1 The minimum value $\gamma_{J}$ of the functional $J(\cdot)$ is finite if and only if $b \in \mathcal{R}(\sqrt{A})$. In addition, let $b=\sqrt{A} d$, with $d \in D(\sqrt{A})$, it holds that

$$
\gamma_{J}=\min _{y \in D(\sqrt{A})}\left(\frac{1}{2}\|\sqrt{A} y\|-(d, \sqrt{A} y)\right)=\min _{z \in H}\left(\frac{1}{2}\|z\|^{2}-(d, z)\right)=-\frac{1}{2}\|d\|^{2} .
$$

Proof. The proof is conducted exactly as was proved Theorem 2.1. The only point that remains to deal with is the explicit value of $\gamma_{J}$ when $b \in \mathcal{R}(\sqrt{A})$. The second equality in (5) is straightforward from the density of $\mathcal{R}(\sqrt{A})$ in $H$. To prove the first equality, we have obviously that

$$
\gamma_{J}=\min _{y \in D(A)}\left(\frac{1}{2}\|\sqrt{A} y\|^{2}-(d, \sqrt{A} y)\right)=\min _{z \in \sqrt{A}(D(A))}\left(\frac{1}{2}\|z\|^{2}-(d, z)\right)
$$

The result is then may be obtained as follows. Because $\sqrt{A}$ is a closed operator, $D(\sqrt{A})$, endowed with the graph norm, denoted $\|\cdot\|_{\sqrt{A}}\left({ }^{1}\right)$, is a Hilbert space. Then, we consider the operator

$$
\begin{aligned}
\sqrt{A}: D(A) \subset D(\sqrt{A}) & \rightarrow D(\sqrt{A}) \\
y & \mapsto \sqrt{A} y,
\end{aligned}
$$

Stating that it is closed is proceeded without any high technicalities. Due to $\mathcal{N}(\sqrt{A})=\{0\}$, we conclude to the density of $\sqrt{A}(D(A))$ in $D(\sqrt{A})$, with respect to $\|\cdot\|_{\sqrt{A}}$, and in particular with the norm of $H$. This completes the proof.

When we are involved in solving equation $A x=b$, the connection between the minimization problem (4) and the least squares method is arisen. The result of Lemma 3.1 is weakened.

Lemma 4.2 Assume that $b \in \mathcal{R}(\sqrt{A})$. Let $\left(x_{n}\right)_{n} \subset D(A)$ be a minimizing sequence of $J(\cdot)$. Then, $\left(\sqrt{A} x_{n}\right)_{n} \subset D(\sqrt{A})$ converges toward $d$ and $\left(A x_{n}\right)_{n} \subset H$ converges weakly toward $b$. Furthermore, if $\left(x_{n}\right)_{n}$ is bounded then $b \in \mathcal{R}(A)$.

Proof. Let $\left(x_{n}\right)_{n} \subset D(A)$ be a minimizing sequence of $J(\cdot)$. That $\left(\sqrt{A} x_{n}\right)_{n} \subset H$ converges toward $d$ can be processed as in Lemma 3.1. The unboundedness of $\sqrt{A}$ does not allow to

\footnotetext{
${ }^{1}$ It is defined by $\|\cdot\|_{\sqrt{A}}=\left(\|\cdot\|^{2}+\|\sqrt{A} \cdot\|^{2}\right)^{1 / 2}$.
} 
directly conclude to the convergence $\left(A x_{n}\right)_{n} \subset H$ toward $b$. We proceed then in a different way. For any $y \in H$ and $t \in \mathbb{R}$, the following decomposition holds

$$
J\left(x_{n}+t y\right)=J\left(x_{n}\right)+t\left(\nabla J\left(x_{n}\right), y\right)+\frac{t^{2}}{2}(A y, y) .
$$

Setting $J\left(x_{n}\right)=\gamma_{J}+\epsilon\left(x_{n}\right)$, we have that $\left(\epsilon\left(x_{n}\right)\right)_{n}$ is non-negative and decays to zero. Inserting in the previous identity, and seen $J\left(x_{n}+t y\right)-\gamma_{J} \geq 0$, we obtain that for $y \neq 0$,

$$
\frac{1}{2}(A y, y)\left(t-\frac{\left(\nabla J\left(x_{n}\right), y\right)}{(A y, y)}\right)^{2}+\epsilon\left(x_{n}\right)-\frac{1}{2} \frac{\left(\nabla J\left(x_{n}\right), y\right)^{2}}{(A y, y)} \geq 0, \quad \forall t \in \mathbb{R} .
$$

That the quadratic polynomial on $t$ has a constant sign yields that

$$
\left(A x_{n}-b, y\right)^{2}=\left(\nabla J\left(x_{n}\right), y\right)^{2} \leq 2 \epsilon\left(x_{n}\right)(A y, y) \leq 2 \epsilon\left(x_{n}\right)\|\sqrt{A} y\|^{2}, \quad \forall y \in H .
$$

This produces

$$
\left|\left(A x_{n}-b, y\right)\right| \leq \sqrt{2 \epsilon\left(x_{n}\right)}\|\sqrt{A} y\|, \quad \forall y \in H .
$$

Passing to the limit provides the weak convergence of $\left(A x_{n}\right)_{n \in \mathbb{N}}$ toward $b$. Now, if $\left(x_{n}\right)_{n \in \mathbb{N}}$ is bounded then it is weakly convergent toward an $x \in H$ (modulo the extraction of a subsequence). That $A$ is a closed operator yields that $x \in D(A)$ and $A x=b$, and then $b \in \mathcal{R}(A)$. The proof is complete.

\section{Fourier Analysis and Weak Picard's Principle}

A particular illustration of the results in Sections 2 and 3 is by a Fourier analysis. So let us assume that the Hilbert space $H$ is separable and that the operator $A$ is compact. As a result, by the Hilbert-Schmidt Theorem, there exists a countable family of eigenvectors and eigenvalues $\left(\zeta_{k}, \gamma_{k}\right)_{k \in \mathbb{N}}$ of $A$ such that $\left(\zeta_{k}\right)_{k \in \mathbb{N}}$ is a Hilbert basis of $H$. The eigenvalues $\left(\gamma_{k}\right)_{k \in \mathbb{N}}$ are all in ] $0,+\infty[$ and tend to zero. Therefore, any $y \in H$ can be expanded along the orthonormal basis $\left(\zeta_{k}\right)_{k \in \mathbb{N}}$

$$
y=\sum_{k \in \mathbb{N}} y_{k} \zeta_{k}, \quad y_{k}=\left(y, \zeta_{k}\right), \quad\|y\|=\sqrt{\sum_{k \in \mathbb{N}} y_{k}^{2}} .
$$

This establishes an isometry between $H$ and the space $\ell^{2}(\mathbb{R})$ (of square summable sequences). Considering the expansion of $A$ on this basis leads to the countable infinite diagonal matrix $\operatorname{diag}\left(\gamma_{k}\right)_{k \in \mathbb{N}} \in \mathcal{M}_{\mathbb{N}}(\mathbb{R})$. Similarly, we associate to the $b$ the countable infinite vector $\left(b_{k}\right)_{k \in \mathbb{N}} \in$ $\mathbb{R}^{\mathbb{N}}$. Looking closely at the functional $J(\cdot)$, yields that either $b_{k}$ goes to zero sufficiently faster than $\gamma_{k}$ and the optimization problem (1) has only one solution, or we have not existence for that problem (the formal solution $x=\left(x_{k}\right)_{k \in \mathbb{N}}=\left(b_{k} / \gamma_{k}\right)_{k \in \mathbb{N}}$ blows up). This is the Picard principle applied to the ill-posed optimization problem or to the equation $A x=b$ (see [14]). Actually, the Picard principle for the existence in $H$ of the argument of the minimum for (1) precisely reads as

$$
\sum_{k \in \mathbb{N}}\left(x_{k}\right)^{2}=\sum_{k \in \mathbb{N}}\left(\frac{b_{k}}{\gamma_{k}}\right)^{2}<+\infty
$$


The event that it is not fulfilled, when $b \notin \mathcal{R}(A)$, compels one rather to handle minimizing sequences. The truncated sum $x^{n}=\sum_{k \leq n} x_{k} \zeta_{k}$ provides the most natural of them.

Now, we intend to exhibit spurious modes generated by the least squares methods. We need, then, to redisplay the energy functional in terms of the Fourier coefficients,

$$
J(y)=\frac{1}{2} \sum_{k \in \mathbb{N}} \gamma_{k}\left(y_{k}\right)^{2}-\sum_{k \in \mathbb{N}} b_{k} y_{k}, \quad \forall y \in H .
$$

Assume that $b \in \mathcal{R}(\sqrt{A})$. A direct computation of the minimum of $J(\cdot)$ yields that

$$
\gamma_{J}=-\frac{1}{2} \sum_{k \in \mathbb{N}} \frac{\left(b_{k}\right)^{2}}{\gamma_{k}}>-\infty .
$$

Let $x^{n}$ be defined by

$$
x^{n}=\sum_{k \leq n-1} \frac{b_{k}}{\gamma_{k}} \zeta_{k}+\frac{1}{\sqrt{\gamma_{n}}} \zeta_{n} .
$$

The sequence $\left(x^{n}\right)_{n \in \mathbb{N}}$ obviously blows up. Again by some explicit calculations we obtain that

$$
\left(\nabla J\left(x^{n}\right), J\left(x^{n}\right)\right)=\left(\sqrt{\gamma_{n}} \zeta_{n}-\sum_{k \geq n} b_{k} \zeta_{k}, \frac{1}{2}-\frac{1}{2} \sum_{k \leq n-1} \frac{\left(b_{k}\right)^{2}}{\gamma_{k}}\right) \in H \times \mathbb{R} .
$$

We deduce that $\left(\nabla J\left(x^{n}\right)\right)_{n}$ tends to zero, $\left(x^{n}\right)_{n}$ is hence a minimizing sequence of the least squares functional whereas the limit of $\left(J\left(x^{n}\right)\right)_{n}$ does not coincide with the infimum $\gamma_{J}$, but with $\gamma_{J}+1 / 2$.

Remark 5.1 Contrary to the least squares problem, the minimizing procedure of the energy function $J(\cdot)$ acts like a filter of the oscillating modes, which appear as "spurious modes" (they could hardly contain any useful information).

Remark 5.2 We infer from (8) that a necessary and sufficient condition for $J(\cdot)$ to have a least-squares form, or for $\gamma_{J}$ to be finite, is that

$$
\sum_{k \in \mathbb{N}} \frac{\left(b_{k}\right)^{2}}{\gamma_{k}}<\infty,
$$

which may be considered as a weak Picard condition.

\section{Some Examples}

After discussing an abstract case coming from the least-squares, we exhibit some examples picked out from the optimal control and inverse data completion problems.

We first need some tools related to the Sobolev spaces. Then, let $\Omega$ be a regular domain in $\mathbb{R}^{m}, m \geq 1$ and $\boldsymbol{x}$ denote the generic point of $\Omega$. The Lebesgue space of square integrable functions $L^{2}(\Omega)$ is endowed with the natural inner product $(\cdot, \cdot)_{L^{2}(\Omega)}$; the associated norm is 
$\|\cdot\|_{L^{2}(\Omega)}$. The Sobolev space $H^{1}(\Omega)$ (resp. $H^{2}(\Omega)$ ) involves all the functions that are in $L^{2}(\Omega)$ so as their first (resp. second) order derivatives. It is provided with the norm $\|\cdot\|_{H^{1}(\Omega)}$ and the semi-norm is denoted by $|\cdot|_{H^{1}(\Omega)}$. For any portion of the boundary $\Upsilon \subset \partial \Omega$, the space $H_{0}^{1}(\Omega, \Upsilon)$ contains all the functions of $H^{1}(\Omega)$ that vanish on $\Upsilon$. When $\Upsilon=\partial \Omega$, we simply write $H_{0}^{1}(\Omega)$ and $H^{-1}(\Omega)$ is the dual space of it. The space $H^{1 / 2}(\Upsilon)$ is the set of the traces over $\Upsilon$ of all the functions of $H^{1}(\Omega)$. The notation $H^{-1 / 2}(\Upsilon)$ is for the topological dual space of $H^{1 / 2}(\Upsilon)$ and $\langle\cdot, \cdot\rangle_{1 / 2, \Upsilon}$ stands for the duality between both spaces. We refer to [1] for a detailed study of the fractionary Sobolev spaces.

\subsection{An Abstract Example}

Let $T$ be a bounded linear operator on $H$ such that zero is an accumulation point of its spec$\operatorname{trum}\left({ }^{2}\right)$. We may assume that $\mathcal{N}(T)=\{0\}$ without loss of generality. The operator $T^{*} T$ is symmetric, semi-definite with an unbounded inverse. Assume $b \in H$, and consider the quadratic functional

$$
J(y)=\frac{1}{2}\left(T^{*} T y, y\right)-(b, y)=\frac{1}{2}\|T y\|^{2}-(b, y) .
$$

The following lemma provides the condition for $J(\cdot)$ to be a least-squares function of the form

$$
\frac{1}{2}\|T y-d\|^{2}-\frac{1}{2}\|d\|^{2} .
$$

Lemma 6.1 The quadratic function $J(\cdot)$ given by (9) is a least-squares function of the particular form (10) if and only if it is bounded from below.

Proof. $i$. - It is currently known that $b=T^{*} d$, for some $d \in H(d$ can be selected in $\overline{\mathcal{R}(T)})$ yields that

$$
J(y)=\frac{1}{2}\|T y-d\|^{2}-\frac{1}{2}\|d\|^{2},
$$

and the minimum value of $J(\cdot)$ is $\gamma_{J}=-\frac{1}{2}\|d\|^{2}$.

ii.- Conversely if the minimum value $\gamma_{J}$ of $J(\cdot)$ exists, using Theorem 2.1 we obtain that $b \in \mathcal{R}\left(\sqrt{T^{*} T}\right)$. The proof is achieved because of the equality $\mathcal{R}\left(T^{*}\right)=\mathcal{R}\left(\sqrt{T^{*} T}\right)$ (see [16]) ${ }^{3}$ ).

Remark 6.1 The difference between Lemma 6.1 and Corollary 2.4 is that the form of the least squares functions in Lemma 6.1 is fixed in advance to (10). The least-squares function given in Corollary 2.4 is different from (10), and is rather provided by

$$
\frac{1}{2}\left\|\sqrt{T^{*} T} y-d^{\prime}\right\|^{2}-\frac{1}{2}\left\|d^{\prime}\right\|^{2} .
$$

\footnotetext{
${ }^{2}$ Equation $T y=d$ is then ill-posed.

${ }^{3}$ Actually, a consequence of $i$. and Theorem 2.1 is that $\mathcal{R}\left(T^{*}\right) \subset \mathcal{R}\left(\sqrt{T^{*} T}\right)$. A quick proof of $\mathcal{R}\left(\sqrt{T^{*} T}\right) \subset$ $\mathcal{R}\left(T^{*}\right)$ is given as follows. Let $x \in H$. Since $\mathcal{R}(T) \subset \mathcal{R}\left(\sqrt{T T^{*}}\right)$, we have that $T x=\sqrt{T T^{*}} z$ for some $z \in H$. Since $T^{*}\left(\sqrt{T T^{*}}\right)=\left(\sqrt{T^{*} T}\right) T^{*}$, we obtain that $\sqrt{T^{*} T} x=T^{*} z$.
} 
Remark 6.2 The distinction between the data $b$ that are in $\mathcal{R}\left(A^{*}\right)$ and those that are not in there has an important effect on the regularization procedures one may use for the stabilization of the computations for the ill-posed optimization problem. Indeed, when $b=A^{*} d$, a general perturbation $(\delta b)\left(\notin \mathcal{R}\left(A^{*}\right)\right)$ has a worse incidence on the computations than a particular perturbation of the form $(\delta b)=A^{*}(\delta d)$ (see $\left.[16,14]\right)$.

\subsection{An Optimal Control Problem}

We are interested in a distributed control problem associated with an elliptic partial differential equation set in $\mathbb{R}^{m}$ (see $[22,11]$ ). For a given $g \in L^{2}\left(\mathbb{R}^{m}\right)$, denote by $v\left(=v_{g}\right) \in H^{2}\left(\mathbb{R}^{m}\right)$ the unique solution of

$$
v-\Delta v=g, \quad \text { in } \mathbb{R}^{m} .
$$

Let $\varphi \in H^{-2}\left(\mathbb{R}^{m}\right)$ be fixed. The optimal control deals with the problem of finding a control $g \in L^{2}\left(\mathbb{R}^{m}\right)$ minimizing the cost function

$$
\min _{g \in L^{2}\left(\mathbb{R}^{m}\right)} J(g)=\min _{g \in L^{2}\left(\mathbb{R}^{m}\right)}\left(\frac{1}{2} \int_{\mathbb{R}^{m}}\left[\left(v_{g}\right)^{2}+\beta\left(\nabla v_{g}\right)^{2}\right] d \boldsymbol{x}-\int_{\mathbb{R}^{m}} \varphi v_{g} d \boldsymbol{x}\right)=\gamma_{J} .
$$

$\beta$ is a positive real-number and we make a notation abuse replacing the duality between $H^{2}\left(\mathbb{R}^{m}\right)$ and $H^{-2}\left(\mathbb{R}^{m}\right)$ by an integral. A comprehensive analysis of this optimal control problem can be easily undertaken in the phase space. We have, therefore, to translate both the functional $J(\cdot)$ and equation (11) in terms of the Fourier transform. Let $\hat{g} \in L^{2}\left(\mathbb{R}^{m}\right)$ stand for the Fourier transform of $g$. The unique solution $v$ of (11) can be characterized as follows

$$
\hat{v}(\boldsymbol{\xi})=\frac{\hat{g}(\boldsymbol{\xi})}{1+\boldsymbol{\xi}^{2}}, \quad \forall \boldsymbol{\xi} \in \mathbb{R}^{m}
$$

$\boldsymbol{\xi}$ is the Fourier variable. Using these new variables the cost function can be changed into

$$
J(g)=\frac{1}{2} \int_{\mathbb{R}^{m}} \frac{1+\beta \boldsymbol{\xi}^{2}}{\left(1+\boldsymbol{\xi}^{2}\right)^{2}}(\hat{g})^{2} d \boldsymbol{\xi}-\int_{\mathbb{R}^{m}} \frac{\hat{\varphi}}{1+\boldsymbol{\xi}^{2}} \hat{g} d \boldsymbol{\xi},
$$

Now, define the operator $A$ on $L^{2}\left(\mathbb{R}^{m}\right)$, as follows

$$
A h=\frac{\left(1+\beta \boldsymbol{\xi}^{2}\right) h}{\left(1+\boldsymbol{\xi}^{2}\right)^{2}}, \quad \forall h \in L^{2}\left(\mathbb{R}^{m}\right) .
$$

It is readily checked that $A$ is continuous, symmetric and positive-definite. The spectrum of $A$ is purely continuous and is given by $\left[0, \max \left(1, \frac{\beta^{2}}{4(\beta-1)}\right)\right]$ (see [7], for the definition of the continuous spectrum). Problem (12) is then ill-posed and we are interested in knowing whether $J(\cdot)$ is a least-squares function with respect to $g$. We are fitted to the quadratic optimization addressed above. Applying Theorem 2.1, $J(\cdot)$ is bounded from below only if $\frac{\hat{\varphi}}{\left(1+\boldsymbol{\xi}^{2}\right)^{1 / 2}} \in L^{2}\left(\mathbb{R}^{m}\right)$ which means that $\varphi \in H^{-1}\left(\mathbb{R}^{m}\right)$. It can be checked that

$$
\gamma_{J}=-\frac{1}{2}\left\langle\varphi,(1-\beta \Delta)^{-1} \varphi\right\rangle_{H^{-1}\left(\mathbb{R}^{m}\right), H^{1}\left(\mathbb{R}^{m}\right)}
$$

Remark 6.3 Pursuing in the Fourier space, one can establish that the control problem (12) has only one solution if $\varphi \in L^{2}\left(\mathbb{R}^{m}\right)$. The argument of the minimum is such that

$$
\beta g_{*}=\varphi+(\beta-1)(1-\beta \Delta)^{-1}(\varphi) \text {. }
$$


Remark 6.4 In case where $\gamma_{J}$ is finite, Corollary 2.4 tells that may $J(\cdot)$ be put under a leastsquares form. The explicit (least-squares) expression of $J(\cdot)$ can be inferred from the fact that $\varphi \in H^{-1}\left(\mathbb{R}^{m}\right)$ may be written as $\varphi=(1-\beta \Delta) \psi$ with $\psi \in H^{1}\left(\mathbb{R}^{m}\right)$. We obtain then

$$
\begin{aligned}
J(g) & =\frac{1}{2} \int_{\mathbb{R}^{m}}\left[\left(v_{g}-\psi\right)^{2}+\beta\left(\nabla v_{g}\right)^{2}\right] d \boldsymbol{x}-\int_{\mathbb{R}^{m}}\left[\varphi v_{g}+\beta \nabla \psi \nabla v_{g}\right] d \boldsymbol{x} \\
& =\frac{1}{2} \int_{\mathbb{R}^{m}}\left[\left(v_{g}-\psi\right)^{2}+\beta\left(\nabla\left(v_{g}-\psi\right)\right)^{2}\right] d \boldsymbol{x}-\frac{1}{2} \int_{\mathbb{R}^{m}}\left[(\psi)^{2}+\beta \nabla(\psi)^{2}\right] d \boldsymbol{x} .
\end{aligned}
$$

\subsection{The Steklov-Poincaré Formulation of the Data Completion problem}

Let $\Omega$ be a regular bounded domain in $\mathbb{R}^{d}$ with $\boldsymbol{n}$ the unit normal to the boundary $\Gamma=\partial \Omega$ oriented outward. Suppose that $\Omega$ is annular and that $\Gamma$ is split into $\Gamma_{C}$, accessible to measures, and inaccessible $\Gamma_{I}$. They are assumed disjoint. Given a flux $\varphi_{C}$ and a datum $g_{C}$, the data completion problem for the Laplace operator is expressed as a Cauchy problem (see [28, 17]): find $u$ such that

$$
\begin{aligned}
-\Delta u & =0 & & \text { in } \Omega, \\
u & =g_{C} & & \text { on } \Gamma_{C}, \\
\partial_{\boldsymbol{n}} u & =\varphi_{C} & & \text { on } \Gamma_{C} .
\end{aligned}
$$

The goal pursued is to complete the data along $\Gamma_{I}$, so to be able to compute $u$ on the whole domain $\Omega$. The lack of a source term in (13) is only for clarity and are by no way restrictive.

To fit the Cauchy problem into a variational framework, we follow [4]. We need the introduction of some more notations. Assume that $g_{C} \in H^{1 / 2}\left(\Gamma_{C}\right)$ and $\varphi_{C} \in H^{-1 / 2}\left(\Gamma_{C}\right)$. Let $\mu$ be in $H^{1 / 2}\left(\Gamma_{I}\right)$, define $u_{D}\left(\mu, g_{C}\right) \in H^{1}(\Omega)$ subjected to a Dirichlet condition $u_{D}\left(\mu, g_{C}\right)_{\mid \Gamma_{C}}=g_{C}$, $u_{D}\left(\mu, g_{C}\right)_{\mid \Gamma_{I}}=\mu$, and satisfies

$$
\int_{\Omega} \nabla u_{D}\left(\mu, g_{C}\right) \nabla v d \boldsymbol{z}=0, \quad \forall v \in H_{0}^{1}(\Omega)
$$

Then, $u_{N}\left(\mu, \varphi_{C}\right) \in H^{1}(\Omega)$ is such that $u_{N}\left(\mu, \varphi_{C}\right)_{\mid \Gamma_{I}}=\mu$, and

$$
\int_{\Omega} \nabla u_{N}\left(\mu, \varphi_{C}\right) \nabla v d \boldsymbol{z}=\left\langle\varphi_{C}, v\right\rangle_{\frac{1}{2}, \Gamma_{C}}, \quad \forall v \in H_{0}^{1}\left(\Omega, \Gamma_{I}\right) .
$$

From now on, we write $u_{N}(\mu)$ instead $u_{N}(\mu, 0)$ and $\breve{u}_{N}\left(\varphi_{C}\right)$ instead of $u_{N}\left(0, \varphi_{C}\right)$ and similar notation abuses are made for $u_{D}$.

We consider the bilinear form $s(\cdot, \cdot)$ on $H^{1 / 2}\left(\Gamma_{I}\right) \times H^{1 / 2}\left(\Gamma_{I}\right)$ and the linear form $\ell(\cdot)$ on $H^{1 / 2}\left(\Gamma_{I}\right)$ defined to be: $\forall \lambda, \mu \in H^{1 / 2}\left(\Gamma_{I}\right)$,

$$
\begin{aligned}
& s(\lambda, \mu)=\int_{\Omega} \nabla u_{D}(\lambda) \nabla u_{D}(\mu) d \boldsymbol{z}-\int_{\Omega} \nabla u_{N}(\lambda) \nabla u_{N}(\mu) d \boldsymbol{z}, \\
& \ell(\mu)=-\int_{\Omega} \nabla \breve{u}_{D}\left(g_{C}\right) \nabla u_{D}(\mu) d \boldsymbol{z}-\left\langle\varphi_{C}, u_{N}(\mu)\right\rangle_{\frac{1}{2}, \Gamma_{C}} .
\end{aligned}
$$


The symmetry and the semi-definiteness of $s(\cdot, \cdot)$ are stated in $[4$, Lemme 3.3].

When existence occurs, the solution of problem (13-15) is the one whose trace $\lambda$ on $\Gamma_{I}$ satisfies $u=u_{D}\left(\lambda, g_{C}\right)=u_{N}\left(\lambda, \varphi_{C}\right)$ and solves the Steklov-Poincaré problem: find $\lambda \in H^{1 / 2}\left(\Gamma_{I}\right)$ such that

$$
s(\lambda, \mu)=\ell(\mu), \quad \forall \mu \in H^{1 / 2}\left(\Gamma_{I}\right) .
$$

Problem (18) is the variational translation of the equation

$$
\partial_{\boldsymbol{n}} u_{D}\left(\lambda, g_{C}\right)=\partial_{\boldsymbol{n}} u_{N}\left(\lambda, \varphi_{C}\right), \quad \text { on } \Gamma_{I} .
$$

In case where there is a solution $\lambda$, Holmgren theorem tells that $u_{D}\left(\lambda, g_{C}\right)=u_{N}\left(\lambda, \varphi_{C}\right)$ which is the solution of the data completion problem. In [5] is provided a comprehensive explanation of why the ill-posedness of the Cauchy problem (18), the operator associated with $s(\cdot, \cdot)$ turns to be of Hilbert-Schmidt type and all its eigenvalues are clustered around the origin excepted a finite number of them.

Remark 6.5 The bilinear form $s(\cdot, \cdot)$ is composed of two symmetric semi-definite bilinear forms denoted $s_{D}(\cdot, \cdot)$ and $s_{N}(\cdot, \cdot)$. Each of them determines an inner-product on $H^{1 / 2}\left(\Gamma_{I}\right)$ which provides each a norm equivalent to the natural one.

Complying with the requirements of Sections 2 and 3, suggests to consider the space $H^{1 / 2}\left(\Gamma_{I}\right)$, endowed with the inner-product $s_{D}(\cdot, \cdot)$ denoted henceforth by $(\cdot, \cdot)_{s_{D}}$. The associated norm is $\|\cdot\|_{s_{D}}$. Then, let $\lambda \in H^{1 / 2}\left(\Gamma_{I}\right)$, we define $T \lambda \in H^{1 / 2}\left(\Gamma_{I}\right)$ to be the unique solution of the elliptic problem

$$
s_{D}(T \lambda, \mu)=s_{N}(\lambda, \mu), \quad \forall \mu \in H^{1 / 2}\left(\Gamma_{I}\right) .
$$

$T$ is symmetric on $H^{1 / 2}\left(\Gamma_{I}\right)$ (when endowed with the inner-product $(\cdot, \cdot)_{s_{D}}$ ). Next, let us consider $f \in H^{1 / 2}\left(\Gamma_{I}\right)$ as the unique solution of

$$
s_{D}(f, \mu)=\ell(\mu), \quad \forall \mu \in H^{1 / 2}\left(\Gamma_{I}\right) .
$$

Setting $A=(I-T)$, problem (18) can be reformulated as follows: find $\lambda \in H^{1 / 2}\left(\Gamma_{I}\right)$ such that

$$
A \lambda=f, \quad \text { in } H^{1 / 2}\left(\Gamma_{I}\right) .
$$

The operator $A$ is continuous symmetric, semi-positive and compact with $\mathcal{N}(A)=\{0\}$ (see $[4$, Theorem 3.1, Lemma 3.3]). The generic situation for $f$ is not to belong to $\mathcal{R}(A)$. To cope with equation (19), one can use the least squares method or the minimization of the energy functional. Notice that: $\forall \mu \in H^{1 / 2}\left(\Gamma_{I}\right)$

$$
\begin{aligned}
J(\mu) & =\frac{1}{2}(A \mu, \mu)_{s_{D}}-(f, \mu)_{s_{D}}=\frac{1}{2} s(\mu, \mu)-\ell(\mu) . \\
L(\mu) & =\frac{1}{2}\|A \mu-f\|_{s_{D}}^{2}=\frac{1}{2} \sup _{\chi \in H^{1 / 2}\left(\Gamma_{I}\right)}\left(\frac{s(\lambda, \chi)-\ell(\chi)}{\|\chi\|_{s_{D}}}\right)^{2}
\end{aligned}
$$


In $[4$, Lemma 4.2$]$ is checked that $J(\cdot)$ is bounded from below and its minimum value is given by (see [4, Lemma 4.3])

$$
\gamma_{J}=-\frac{1}{2}\left|\breve{u}_{D}\left(g_{C}\right)-\breve{u}_{N}\left(\varphi_{C}\right)\right|_{H^{1}(\Omega)}^{2} .
$$

Applying Theorem 2.1 yields that $f \in \mathcal{R}(\sqrt{A})$ for any Cauchy data $\left(g_{C}, \varphi_{C}\right) \in H^{1 / 2}\left(\Gamma_{C}\right) \times$ $H^{-1 / 2}\left(\Gamma_{C}\right)$.

When a numerical simulation of the Cauchy solution $u$ is pursued, a performing methodology is to approximate equation (19) with the incomplete boundary unknown $\lambda$. The fact that $f$ is more regular than expected, even when the data $\left(g_{C}, \varphi_{C}\right)$ are polluted, together with the explicit knowledge of $\gamma_{J}$, have important effects on the regularization strategies such as the Lavrentiev method, necessary in computing the solution for ill-posed problems. These issues will be focussed on in the future.

\subsection{Ill-posed Integral Equations}

Let be given a Mercer kernel $G \in L^{2}(\Omega \times \Omega)$ (see [24]) which means that $G(x, y)=G(y, x)>0$, $\forall(x, y) \in \Omega \times \Omega$ and

$$
\int_{\Omega} \int_{\Omega} G(x, y) \psi(x) \psi(y) d x d y \geq 0, \quad \forall \psi \in L^{2}(\Omega) .
$$

Define the linear operator in $L^{2}(\Omega)$ by

$$
A \psi(x)=\int_{\Omega} G(x, y) \psi(y) d x, \quad \forall \psi \in L^{2}(\Omega) .
$$

It is continuous, symmetric, semi-definite and belongs to the class of Hilbert-Schmidt operators; it is then compact (see [7, 1]). In addition, if $\mathcal{N}(A)=\{0\}$ (which is assumed henceforth), then $A$ is of non-closed and dense range, that is $\overline{\mathcal{R}(A)}=H$.

Let $f$ be in $L^{2}(\Omega)$. So many problems are reducible to solve equations similar to: find $\varphi \in L^{2}(\Omega)$ satisfying

$$
A \varphi=f, \quad \text { in } L^{2}(\Omega) .
$$

Such an equation arises in image processing, in signal identification, in computerized tomography, in geophysics inspection, in remote sensing and in so many other industrial or biomedical processes (see [19, 20]). The generic situation is that $f \notin \mathcal{R}(A)$ and $A$ has not a continuous inverse. Small changes in the data $f$ can cause arbitrarily large changes in the results. Problem (20) is thus ill-posed and the ill-posedness degree of it is narrowly related to the regularity of the Kernel $G(\cdot, \cdot)$. The more it is regular the more severe is the ill-posedness (see [15]).

When exact solutions fail for equation (20), practitioners resort to some approximating methods such that least squares procedure or the minimization of the quadratic energy functional. 
Because of the spurious modes tolerated by the least-squares, we prefer to rather focus on the minimization of the functional

$$
J(\psi)=\frac{1}{2}(A \psi, \psi)_{L^{2}(\Omega)}-(f, \psi)_{L^{2}(\Omega)}, \quad \forall \psi \in L^{2}(\Omega) .
$$

The operator $A$ being in the class of Hilbert-Schmidt operators is then compact with a nonclosed range. We are therefore involved in an ill-posed semi-definite quadratic optimization. Applying the results of Section 2 results in

Lemma 6.2 The function $J(\cdot)$ is bounded from below $\left(\gamma_{J}\right.$ is finite) if

$$
\left(\int_{\Omega} f(x) \psi(x) d x\right)^{2} \leq \int_{\Omega} \int_{\Omega} G(x, y) \psi(x) \psi(y) d x d y, \quad \forall \psi \in L^{2}(\Omega) .
$$

Proof. On account of Lemma 2.3, estimate (21) tells that $f \in R(\sqrt{A})$. We conclude by Theorem 2.1.

Remark 6.6 The integral equation (20) has a unique solution in $L^{2}(\Omega)$ means that $f \in \mathcal{R}(A)$ and then

$$
\left(\int_{\Omega} f(x) \psi(x) d x\right)^{2} \leq C \int_{\Omega} \int_{\Omega} \int_{\Omega} G(x, y) G(x, z) \psi(y) \psi(z) d x d y d z, \quad \forall \psi \in L^{2}(\Omega) .
$$

Remark 6.7 Thanks to Mercer's Theorem (see [2]), there exists an orthonormal basis $\left(\chi_{k}\right)_{k \in \mathbb{N}}$ and a sequence of nonnegative real-numbers $\left(\gamma_{k}\right)_{k \in \mathbb{N}}$ such that

$$
G(x, y)=\sum_{k \in \mathbb{N}} \gamma_{k} \chi_{k}(x) \chi_{k}(y), \quad \forall x, y \in \Omega
$$

The functional $J(\cdot)$ can then be obtained through the components on the basis $\left(\chi_{k}\right)_{k \in \mathbb{N}}$ of any $\psi \in$ $L^{2}(\Omega)$,

$$
J(\psi)=\frac{1}{2} \sum_{k \in \mathbb{N}} \gamma_{k}\left(\psi_{k}\right)^{2}-\sum_{k \in \mathbb{N}} f_{k} \psi_{k} .
$$

$\left(\psi_{k}\right)_{k \in \mathbb{N}}$ are the components of $\psi$ on the basis $\left(\chi_{k}\right)_{k \in \mathbb{N}}$. We are exactly in the framework of Section 4. Estimates (22) and (21) can then be translated respectively into

$$
\begin{gathered}
\sum_{k \in \mathbb{N}}\left(\frac{f_{k}}{\gamma_{k}}\right)^{2}<+\infty \\
\sum_{k \in \mathbb{N}} \frac{\left(f_{k}\right)^{2}}{\gamma_{k}}<+\infty
\end{gathered}
$$

Inequality (23) is the Picard condition for the solution of (20) to exist while inequality (24) is the weak form of the Picard condition, for the minimum value $\gamma_{J}$ to be finite.

Remark 6.8 Here again, a real-life example of problem (20), is picked from data completion (13)-(15), where the data is more regular than expected. To avoid working in $H^{1 / 2}\left(\Gamma_{I}\right)$, which requires more technicalities, we consider the normal derivative $\lambda=\partial_{n} u$ as the main 
unknown rather than finding the trace of $u$ on $\Gamma_{I}$. This variable solves an integral equation constructed from the following Green functions. Let $\boldsymbol{z} \in \Omega$ and $\delta_{\boldsymbol{z}}$ is the Dirac distribution centered at $\boldsymbol{z}$. Consider the Green functions $G_{D}$ and $G_{N}$ solutions of the boundary value problems: find $G_{D}(z, \cdot)$ such that

$$
\begin{aligned}
-\Delta G_{D}(\boldsymbol{z}, \cdot) & =\delta_{\boldsymbol{z}} & & \text { in } \Omega, \\
G_{D}(\boldsymbol{z}, \cdot) & =0 & & \text { on } \Gamma_{C}, \\
\partial_{\boldsymbol{n}} G_{D}(\boldsymbol{z}, \cdot) & =0 & & \text { on } \Gamma_{I},
\end{aligned}
$$

and: find $G_{N}(\boldsymbol{z}, \cdot)$ such that

$$
\begin{aligned}
-\Delta G_{N}(\boldsymbol{z}, \cdot) & =\delta_{\boldsymbol{z}} & & \text { in } \Omega, \\
\partial_{\boldsymbol{n}} G_{N}(\boldsymbol{z}, \cdot) & =0 & & \text { on } \Gamma_{C}, \\
\partial_{\boldsymbol{n}} G_{N}(\boldsymbol{z}, \cdot) & =0 & & \text { on } \Gamma_{I} .
\end{aligned}
$$

Both kernels do exist in $L^{2}(\Omega)$ (see e.g. [7]). We define

$$
\begin{aligned}
\mathcal{S} \mu(\boldsymbol{z}) & =\int_{\Gamma_{I}}\left(G_{N}-G_{D}\right)(\boldsymbol{z}, \boldsymbol{y}) \mu(\boldsymbol{y}) d \gamma_{y}, \quad \forall \boldsymbol{z} \in \Omega . \\
\ell(\boldsymbol{z}) & =-\int_{\Gamma_{C}} G_{N}(\boldsymbol{z}, \boldsymbol{y}) \varphi_{C}(\boldsymbol{y}) d \gamma_{y}-\int_{\Gamma_{C}} \partial_{n} G_{D}(\boldsymbol{z}, \boldsymbol{y}) g_{C}(\boldsymbol{y}) d \gamma_{y}, \quad \forall \boldsymbol{z} \in \Omega .
\end{aligned}
$$

The operator $\mathcal{S}$ naturally maps $H^{-1 / 2}\left(\Gamma_{I}\right)$ into $H^{1 / 2}\left(\Gamma_{I}\right)$ and $\ell \in H^{1 / 2}\left(\Gamma_{I}\right)$. Restricted to $L^{2}\left(\Gamma_{I}\right)$, $\mathcal{S}$ defines a Hilbert-Schmidt operator (see [5]). Moreover, it is symmetric and semi-definite with $N(\mathcal{S})=\{0\}$. It is then a Mercer kernel. Thanks to the elliptic regularity, when existence is guaranteed $\left({ }^{4}\right)$ the solution $\lambda$ turns to be in $L^{2}\left(\Gamma_{I}\right)$ and satisfies

$$
\mathcal{S} \lambda=\ell
$$

It is also the argument of the minimum of the function $J(\cdot)$, defined as follows,

$$
J(\mu)=\frac{1}{2}(\mathcal{S} \mu, \mu)_{L^{2}\left(\Gamma_{I}\right)}-(\ell, \mu)_{L^{2}\left(\Gamma_{I}\right)}, \quad \forall \mu \in L^{2}\left(\Gamma_{I}\right) .
$$

In [5] is proved that, whatever $\left(g_{C}, \varphi_{C}\right)$ are in $H^{1 / 2}\left(\Gamma_{I}\right) \times H^{-1 / 2}\left(\Gamma_{I}\right)$, the functional $J(\cdot)$ is bounded from below which means that $\ell \in \mathcal{R}(\sqrt{\mathcal{S}})$.

\section{Conclusion}

Ill posed quadratic optimization arises, for example, in optimal control and in inverse problems such as the data completion. The finiteness of the minimal value of the cost function is guaranteed for regular data. As illustrated in the current paper, such a regularity is naturally fulfilled in many problems. We emphasize on the fact that bounding the cost function from below provides valuable informations on the smoothness of the data which turns to be useful for the regularization strategies such as the Lavrentiev or the Mann methods (see $[21,23,10]$ ).

\footnotetext{
${ }^{4}$ The data $\left(g_{C}, \varphi_{C}\right)$ are said compatible.
} 


\section{References}

[1] Adams D. A. ; Sobolev Spaces, Academic Press, New-York (1975).

[2] Aronszajn, N. ; Theory of reproducing kernels, Trans. Amer. Math. Soc. 68, pp. 337-404 (1950).

[3] Auslender, A. A. ; Crouzeix, J.-P.- Well-behaved asymptotical convex functions. Analyse non linaire (Perpignan, 1987). Ann. Inst. H. Poincaré Anal. Non Linéaire, 6, pp. 101-121 (1989).

[4] Ben Belgacem, F. ; El Fekih H., (2005) On Cauchy's Problem. I. A Variational SteklovPoincaré Theory, Inverse Problems, 21, 1915-1936 (2005).

[5] Ben Belgacem, F. ; Why is the Cauchy's Problem Severely Ill-posed? Inverse Problems, 23, 823-836 (2007).

[6] Bonnans, J.F. ; Gilbert, J. Ch. ; Lemaréchal, C. ; Sagastizbal, C. ; Optimisation numrique, Springer-Verlag (1997).

[7] Dautray, R. ; Lions, J.-L. ; Mathematical analysis and numerical methods for science and technology. Volume 2. Functional and variational methods. With the collaboration of M. Artola, M. Authier, Ph. Bénilan, M. Cessenat, J.-M. Combes, H. Lanchon, B. Mercier, Cl. Wild and Cl. Zuily. Translated from the French by Ian N. Sneddon. Springer-Verlag, Berlin, (1988).

[8] Dunford, N., Schwartz, J. T. ; Linear Operators, Part I, Interscience. New York (1958).

[9] Ekland, I. ; Temam, R. ; Analyse convexe et problèmes variationnels, Dunod-GauthierVillars, 1973.

[10] Engl, H. W. ; Leitão, A. ; A Mann iterative regularization method for elliptic Cauchy problems. Numer. Funct. Anal. Optim., 22, pp. 861-884 (2001).

[11] Fursikov, A. V. - Optimal control of distributed systems: theory and applications Providence, RI: American Mathematical Society (2000).

[12] Gould, N. I. M. ; Toint, Ph. L. ; A Quadratic Programming Bibliography.Numerical Analysis Group Internal Report 2000-1 Rutherford Appleton Laboratory, Chilton, England.

[13] Hadamard, J. ; Lectures on Cauchy's problem in linear partial differential equation (New York: Dover) (1953).

[14] Hansen, P. C. ; Rank-deficient and discrete ill-posed problems. Numerical aspects of linear inversion. SIAM Monographs on Mathematical Modeling and Computation. Society for Industrial and Applied Mathematics (SIAM), Philadelphia, PA, (1998).

[15] Hille E., Tamarkin J. D. ; On the Characteristic Values of Linear Integral Equations. Proceedings of the National Academy of Sciences, (1928). 
[16] Hohage, T. ; Lecture Notes in Inverse Problems, University of Göttingen, (2002).

[17] Isakov, V.- Inverse Problems for Partial Differential Equations. Applied Mathematical siences 127, Springer-Verlag, New-York (1998).

[18] Kaplan, A. ; Tikhachke, R. ; Stable methods for ill-posed variational problems : Prox regularization of elliptic variational inequalities and semi finite problems, Akademie Verlag, Berlin (1994).

[19] Kirsch, A. ; An introduction to the mathematical theory of inverse problems. Applied Mathematical Sciences, 120. Springer-Verlag, New York, (1996).

[20] Kress R. ; Linear Integral Equations. Applied Mathematical Sciences. Springer-Verlag, Berlin Heidelberg (1989).

[21] Lavrentiev, M. M. ; Some Improperly Posed Problems of Mathematical Physics. New York: Springer-Verlag (1967).

[22] Lions, J.-L. ; Contrôle des systèmes distribués singuliers, Dunod, (1983).

[23] Nair, M. T. ; Tautenhahn, U. ; Lavrentiev regularization for linear ill-posed problems under general source conditions. Z. Anal. Anwendungen 23, 167-185 (2004)

[24] Mercer, J. ; Functions of positive and negative type and their connection with the theory of integral equations., Philos. Trans. Roy. Soc. London, 1909.

[25] Rasmussen, K. E. ; Williams, C. K. I. ; Gaussian Processes for Machine Learning. MIT Press, (2006).

[26] Riesz, F. ; Nagy, U. ; Leçons d'analyse fonctionnelle. Paris: Gauthier-Villars (1972).

[27] Saad, Y. ; Iterative Methods for Sparse Linear Systems. Second Edition, SIAM (2003).

[28] Tarkhanov, N. N. ; The Cauchy problem for solutions of elliptic equations. Mathematical Topics, 7, Akademie Verlag, Berlin, (1995).

[29] Tikhonov A. N. ; Arsenin V. Y. Solution to Ill-posed Problems (New York: Winston-Wiley) (1977).

[30] Wolkowicz, H. ; Saigal, R. ; Vandenberghe, L. ; Handbook of semidefinite programming. Theory, Algorithms, and Applications. Dordrecht, Netherlands: Kluwer (2000). 